\title{
Scleroderma of geriatric age and scleroderma-like paraneoplastic syndrome - description of two cases
}

\author{
Magdalena Marek ${ }^{1}$, Robert Rudny ${ }^{2}$ \\ ${ }^{1}$ Department of Rheumatology, Specialist Hospital, Kościerzyna, Poland \\ ${ }^{2}$ Branch I, Jadwiga Titz-Kosko Voivodeship Rheumatology Complex, Sopot, Poland
}

\begin{abstract}
Systemic sclerosis (Ssc) is an autoimmune connective tissue disease of unknown origin, characterized by progressive fibrosis of the skin and internal organs.

Immune reactions taking part in Ssc pathogenesis may contribute to cancer development; therefore patients with risk factors for this disease require observation for a neoplastic process. On the other hand, symptoms of Ssc may be a mask of various cancers. Differentiating between the idiopathic form of Ssc and scleroderma-like paraneoplastic syndrome often causes a lot of difficulties.

The article presents two cases of Ssc at the beginning of the disease after 60 years of age. The first case was diagnosed as Ssc, whereas in the second case the defined diagnosis was scleroderma-like syndrome in the course of colorectal cancer. This paper presents an analysis of differential diagnostic procedures which were performed and led to the final diagnosis, mentions types of cancers co-occurring with Ssc and suggests a screening scheme for cancer development in patients with a diagnosis of Ssc.
\end{abstract}

Key words: cancer, scleroderma, paraneoplastic syndrome.

\section{Introduction}

Systemic sclerosis (Ssc) is a connective tissue disease with average incidence up to $0.002 \%$. In Poland there are about 10 thousand people suffering from Ssc. The disease most often develops in the 30s-50s and predominates among women $[1,2]$. It is likely that in most cases, the symptoms of Ssc mimic a rheumatological mask of cancer. The pathomechanism of paraneoplastic syndrome has not been completely discovered yet. One of many theories suggests that cancer cells may induce synthesis of a substance which leads to fibrosis of the skin and internal organs [1, 3].

Many studies confirm that patients suffering from Ssc, especially those over 50 years old, have statistically higher risk of cancer development. It is possible that predisposed patients may develop two separate diseases with a similar pathogenic mechanism [3]. Therefore we suggest a screening scheme for patients diagnosed with Ssc.

We analyzed two cases of patients with the first symptoms suggesting Ssc at the age of 75 and 60 . The extensive examination performed helped to diagnose the first patient with idiopathic Ssc without co-occurring neoplasm, while the second patient was diagnosed with scleroderma-like paraneoplastic syndrome (SLPS) in the course of colon cancer.

\section{Cases description}

\section{Case 1}

A 78-year-old patient was admitted to the Rheumatic Diseases Hospital in Sopot in July 2011, due to Raynaud's phenomenon observed for about 3 years, progressive skin thickening, difficulty in swallowing, poor exercise tolerance and weight loss (about $20 \mathrm{~kg}$ within

\section{Address for correspondence:}

Magdalena Marek, Department of Rheumatology, Specialist Hospital, Piechowskiego 36, 83-400 Kościerzyna, Poland,

e-mail: magdalena.marek@googlemail.com

Submitted: 18.04.2016; Accepted: 28.04.2016 
2 years). The patient suffered from hypertension, chronic renal failure, sustained atrial fibrillation and chronic ischaemic heart disease; in 1988 the patient had a heart attack.

Within a 2-year period prior to admission an extensive examination was performed: computed tomography $(\mathrm{CT})$ of the abdominal cavity, abdominal cavity and head magnetic resonance (MR), chest high-resolution computed tomography (HRCT), and endoscopy of the upper and lower part of the gastrointestinal tract - results excluded a neoplastic process. Chest HRCT revealed interstitial lung fibrosis.

Physical examination revealed fibrosis of the skin of distal parts of upper and lower limbs, chest, face (modified Rodnan skin test was 22), lip atrophy, facial telangiectasias, and small ulcers at the tip of the fingers, and auscultation revealed bilateral crackles at the base of the lung.

Laboratory tests showed: macrocytic anaemia, features of chronic renal failure, high value of erythrocyte sedimentation rate (ESR), and correct level of C-reactive protein (CRP). Blood was sent for immunology testing: results were positive for antinuclear antibodies (titre $1: 640$, speckled pattern), anti SS-A-Ro52, RNP/Sm and Scl70 antibodies (immunoblot test). Hand nailfold capillaroscopy showed abnormalities corresponding to late scleroderma pattern angiopathy (Fig. 1). Echocardiography showed dilated biventricular size, asymmetric interventricular septal hypertrophy, hypokinesis of lateral wall, and high pressure in right ventricle $(80 \mathrm{~mm} \mathrm{Hg}$ ). Based on the clinical picture and additional examinations, Ssc with diffuse scleroderma was diagnosed.

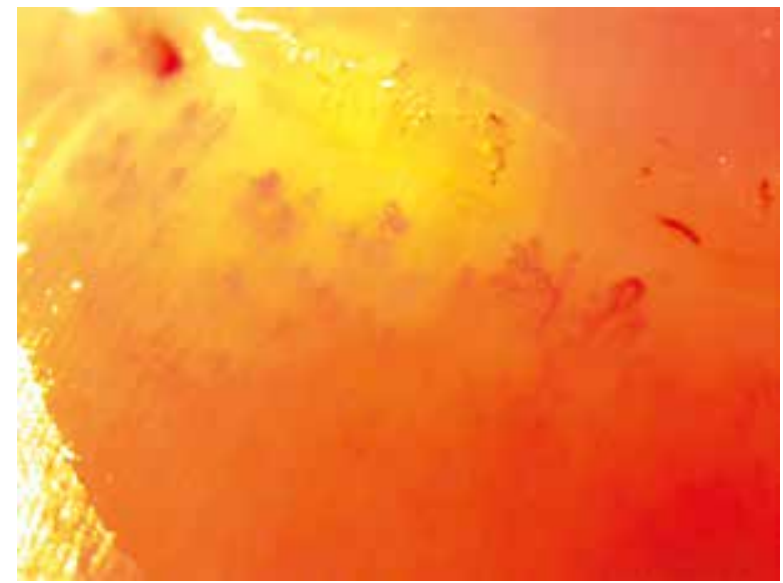

Fig. 1. Nailfold capillaroscopy (magnification $100 \times$ ) - intense desorganisation of the normal capillary array, severe loss of capillaries, avascular areas, features of neoangiogenesis - scleroderma late pattern (authors library).
The patient was consulted in the Clinic of Heart Electrotherapy, where cardiological tests including right heart catheterization were performed and the diagnosis of pulmonary hypertension of mixed etiology was established. The patient was not qualified for treatment with bosentan, so sildenafil was assigned.

After consultation with a nephrologist it was decided to start the therapy with cyclophosphamide in modified doses due to renal failure. In the period from July to September 2011 a total of 1000 mg was administered. The treatment was discontinued because of the patient's poor tolerance manifested by repeated lower respiratory tract infections, loss of appetite, and increasing weakness. The patient continued treatment with sildenafil. Three years after the Ssc diagnosis the patient died. Throughout the entire period of observation the patient was not diagnosed with cancer.

\section{Case 2}

A 59-year-old patient was admitted to the Rheumatic Diseases Hospital in Sopot in August 2012 due to symptoms of hands, forearms and face skin fibrosis without accompanying Raynaud's phenomenon. Apart from pain in the lumbosacral spine, the patient did not report any symptoms. The patient was suffering from chronic hypertension, and he was also a long-term smoker. The physical examination showed fibrosis of the skin (modified Rodnan skin score was 18) and enlarged neck lymph nodes.

Laboratory tests showed slightly increased values of inflammation parameters (ESR - $23 \mathrm{~mm} / \mathrm{h}, \mathrm{CRP}-8.2$ $\mathrm{mg} / \mathrm{l}$ ), immunology tests revealed ANA (titre 1 : 2560,

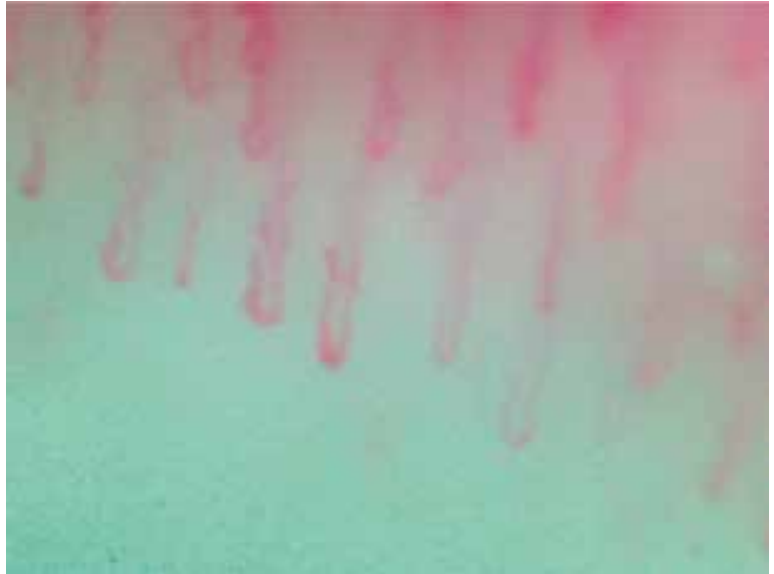

Fig. 2. Nailfold capillaroscopy (magnification $400 \times$ ) - non specific capillaroscopic abnormalities characterised by tortuous and enlarged loops (authors library). 
speckled pattern), and results of immunoblot and scleroderma-specific antibodies were negative.

Hands nailfold capillaroscopy showed non-specific capillary abnormalities which did not meet the criteria for a scleroderma pattern (Fig. 2). Chest X-ray revealed pulmonary hilar enlargement. Chest CT was carried out, where enlarged mediastinal lymph nodes were identified. Mediastinoscopy was performed, a mediastinal lymph node was sampled for histopathological examination, which indicated the presence of cancer cells, and adenocarcinoma was suggested.

In October 2012 the patient experienced severe renal failure involving urinary obstruction of unclear aetiology. Right nephrostomy was performed and improved the patient's state. After further examinations the patient was transferred to the Internal Diseases Ward, where colonoscopy revealed a tumour in the ascending colon. On the basis of histopathological examination the colorectal adenocarcinoma was diagnosed. On the first day after the colonoscopy, symptoms of severe peritonitis and septic shock occurred. In spite of reoperation and treatment in the Intensive Care Unit, the patient died.

\section{Discussion}

Systemic sclerosis is a disease of middle age, most often diagnosed between 30 and 50 years of age. Women according to EUSTAR (EULAR Scleroderma Trials and Research Group) are affected more often and constitute $87 \%$ in the research. It is rather rare to diagnose a patient older than $60[1,2]$. Data from the literature suggest that scleroderma in the elderly occurs more frequently than is assumed but it is not well diagnosed.

The reasons speculated for this are the following:

- elderly people are less often referred for specialist consultation,

- the clinical picture of the disease may be slightly different in this age group, for instance relatively mild skin lesions as well as similarity of Ssc and common elderly chronic disease symptoms [4].

Systemic sclerosis diagnosis after 50 is an unfavourable predictor: a higher death rate and higher risk of cancer diagnosis than with younger patients is observed [5]. In the case of occurrence of Ssc in elderly patients, two possibilities shall be considered: paraneoplastic syndrome or idiopathic scleroderma (in the course of which cancer is very likely to be diagnosed in the following years).

Scleroderma-like paraneoplastic syndrome is a group of symptoms similar to systemic sclerosis which occur in cancer patients [1]. Diagnostics aiming at excluding proliferative disease is extremely difficult when the tumour is asymptomatic. Reasons why this syndrome occur are sophisticated and not entirely discovered. Maybe the expression and presentation of antigens on the surface of tumour cells activates the immune system, which results in production of antibodies that cross-react with the organism tissues, causing their damage and secondary regeneration. However, antigen expression gets much stronger in damaged tissues, strongly stimulates the immune system and closes the chain of reactions resulting in occurrence of the symptoms of immune disease [3]. Specific anomalies in the clinical picture and examination may suggest SLPS early on in the diagnostic process. Especially:

- a short-term break between the diagnosis of both diseases,

- old age of a patient,

- positive family history for cancer,

- patient's exposure to carcinogenesis in medical history,

- asymmetric skin fibrosis,

- no or asymmetric Raynaud's phenomenon,

- lack of abnormalities typical for Ss in capillaroscopy,

- lack of antibodies characteristic for scleroderma,

- general symptoms such as fever and weight loss,

- no response to standard treatment (rather difficult to estimate in the case of scleroderma, since so far no effective medicine has been discovered to stop the progression of the disease),

- disappearance of Ssc symptoms after anticancer treatment and their appearance when the cancer reactivates [6].

The second case was characterized by lack of Raynaud's phenomenon, lack of abnormalities typical for scleroderma in capillaroscopy, and lack of antibodies specific for SSc, which could suggest SLPS.

Many studies have shown higher frequency of cancers in Ssc patients, occurring in 3-7\% of patients. These are most often lung and breast cancers, hematopoietic cancer (mainly non-Hodgkin's lymphoma), skin cancers excluding melanoma, and also bladder and liver cancers. The cancer may be diagnosed just before the symptoms of Ssc occur, at the same time, 2-3 years later or after many years of disease presence $[3,7]$.

Researchers try to explain a common pathogenic mechanism of occurrence of both diseases, Ssc and cancer. The following aspects are taken into consideration: defect in immune monitoring and impaired purification of carcinogens, genetic susceptibility to both malignancy and autoimmune disease, the influence of cytotoxic immunosuppressive therapy, and increased susceptibility to cancer transformation connected with chronic inflammation. Patients with long-term, active interstitial lung fibrosis, longstanding gastro-oesophageal reflux disease and extensive skin fibrosis have higher risk of development of late lung, skin or oesophageal cancers. 
Such excessive proliferation of cells increases the risk of mutation $[3,7,8]$.

Moreover, a correlation between a certain group of antibodies characteristic for Ssc and the cancer risk has been detected. It was observed that the patients with antibodies against RNA polymerase III had a short period of time between diagnosis and occurrence of Ssc symptoms, compared to patients with anti-centromere and topoisomerase I antibodies [3, 9].

We suggest the following screening scheme (used routinely in our practice):

1. Self-inspection of skin moles, UV protection, regular dermatological check-ups along with dermatoscopy, early removal of suspicious moles.

2. Regular lung function tests are recommended as well as chest X-ray or HRCT once a year, especially for smoking patients.

3. Breast self-examination once a month, breast palpation on every visit to the doctor, mammography, ultrasound examination of breasts once a year. Strong oncologic vigilance is recommended especially within the first five years after diagnosis (most cases of breast cancer are diagnosed within 2-3 years of the first occurrence of scleroderma symptoms). Chest skin fibrosis can make palpation difficult, and may impact the interpretation of imaging examination.

Summing up, a correlation between Ssc and cancer is evident. The correlation is multidimensional and not completely clear. It is often impossible to differentiate, especially at an early stage of the disease, between idiopathic SSC and SLPS, and an undetected tumour. Therefore all patients with symptoms of Ssc, especially elderly ones, should be examined for cancer.

The authors declare no conflict of interest.

\section{References}

1. Kowal-Bielecka O, Bielecki M. Twardzina układowa. In: Puszczewicz M (ed.). Reumatologia. Wielka interna. Medical Tribune Polska, Warszawa 2010; 127-141.

2. Sierakowski S, Sierakowska M. Twardzina układowa. In: Szczeklik A (ed.). Choroby wewnętrzne. Vol. II. Medycyna Praktyczna, Kraków 2006

3. Shah A, Rosen A. Cancer and systemic sclerosis: novel insights into pathogenesis and clinical implications. Curr Opin Rheumatol 2011; 23: 530-535.

4. Systemic sclerosis in old age. Br Med J 1979; 2: 1313-1314

5. Zimmermann-Górska I. Reumatologia kliniczna. Wydawnictwo Lekarskie PZWL, Warszawa 2008; 1121.

6. Puszczewicz M, Ociepa-Zawal M. Objawy reumatologiczne w przebiegu chorób nowotworowych. In: Puszczewicz M (ed.) Reumatologia. Wielka interna. Medical Tribune Polska, Warszawa 2010; 502-511.
7. Zhang JQ, Wan YN, Peng WJ, et al. The risk of cancer development in systemic sclerosis-metaanalysis. Cancer Epidemiol 2013; 37: 523-527.

8. Onishi A, Sugiyama D, Kumagai S, Morinobu A. Cancer incidence in systemic sclerosis. Arthritis Rheum 2013; 65: 1913-1921.

9. Shah AA, Rosen A, Hummers L, et al. Close temporal relationship between onset of cancer and scleroderma in patients with RNA polymerase I/II antibodies. Arthritis Rheum 2010; 62: 2787-2795. 